\title{
Fast and accurate modeling of waveguide grating couplers
}

\author{
P. G. Dinesen \\ Department of Optics and Fluid Dynamics, Risø National Laboratory, OFD-128, P.O. Box 49, \\ DK-4000 Roskilde, Denmark \\ J. S. Hesthaven \\ Division of Applied Mathematics, Brown University, Box F, Providence, Rhode Island 02912
}

Received March 16, 2000; revised manuscript received May 30, 2000; accepted June 6, 2000

\begin{abstract}
A boundary variation method for the analysis of both infinite periodic and finite aperiodic waveguide grating couplers in two dimensions is introduced. Based on a previously introduced boundary variation method for the analysis of metallic and transmission gratings [J. Opt. Soc. Am. A 10, 2307, 2551 (1993)], a numerical algorithm suitable for waveguide grating couplers is derived. Examples of the analysis of purely periodic grating couplers are given that illustrate the convergence of the scheme. An analysis of the use of the proposed method for focusing waveguide grating couplers is given, and a comparison with a highly accurate spectral collocation method yields excellent agreement and illustrates the attractiveness of the proposed boundary variation method in terms of speed and achievable accuracy. (C) 2000 Optical Society of America [S0740-3232(00)01709-9]
\end{abstract}

OCIS codes: $050.0050,050.1960,000.4430$.

\section{INTRODUCTION}

In recent years an increasing interest in the design and use of diffraction-based integrated optics has led to a need for fast and accurate numerical methods for the analysis and design of diffraction-dominated structures. The formulation and development of such methods are severely complicated by the rigorous treatment of the vector-field behavior in the resonant regime, where the wavelength of the optical field is comparable with the size of the geometrical features.

For inherently periodic structures of infinite extent, rigorous coupled-wave analysis has been widely used for more than a decade. ${ }^{1}$ However, the need to analyze structures with aperiodic features and of finite extent has led to the need for methods that are capable of dealing with such structures, and the use of the finite-element, ${ }^{2}$ boundary element, ${ }^{3}$ finite-difference time-domain, ${ }^{4,5}$ and spectral collocation ${ }^{6}$ methods has been proposed. The two last-named methods both compute a direct solution of the time-domain vectorial Maxwell equations and are quite general in being adaptable to a wide variety of geometries and physical settings. As the need to model problems of realistic size and complexity becomes more pressing, however, the memory and computational time requirements of such direct volume methods quickly become limiting factors not only for the design process but also for analyses of particular structures.

In this paper we propose to take a different road, guided by the work of Bruno and Reitich. ${ }^{7}$ They have established that solutions to electromagnetic diffraction by a periodic structure depend analytically on the variations of the interface. In other words, diffraction from a peri- odic grating can be determined from knowledge of reflection and refraction at a plane interface. Using this result, Bruno and Reitich proposed a high-order perturbation scheme for finite-size perturbations and successfully used it in modeling diffraction by two- and threedimensional metallic and transmission gratings. ${ }^{8,9}$

We propose a further development that leads to a formulation for the analysis of waveguide grating couplers in which a guided wave in a thin-film waveguide is coupled to free-space radiation through surface relief, as sketched in Fig. 1. Moreover, we extend the analysis to structures of finite extent and demonstrate, by comparison with a highly accurate spectral collocation method, that the method of boundary variation provides surprisingly accurate results for the modeling of radiation from waveguide grating couplers.

The remainder of this paper is organized as follows: In Section 2 we present the modified boundary variation (BV) method as applied to the waveguide grating coupler and discuss the details of the numerical implementation, including postprocessing by free-space integration of the radiated field. In Section 3 we demonstrate the use of the proposed code for the analysis of periodic waveguide grating couplers, and in Section 4 we turn to the analysis of finite-length focusing grating couplers (FGC's) with aperiodic grating functions. We present a comparison of the proposed method with a spectral collocation code to demonstrate the accuracy of the BV method for the modeling of nontrivial waveguide grating couplers. We conclude with a discussion of the superior computational efficiency of the proposed approach and a few remarks on directions of future research. 

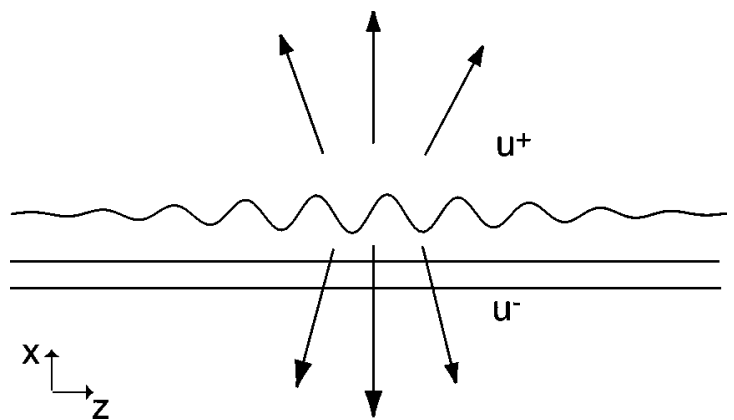

Fig. 1. Thin-film optical waveguide comprising a surface-relief grating for coupling from guided-wave to free-space radiation.

\section{BOUNDARY VARIATION FOR GRATING COUPLERS}

In the following, we shall outline the elements of the proposed algorithm without going into details of the algebra involved in the derivation of the scheme but rather dwell on the properties of a field propagating in a thin-film waveguide. It is an understanding of these properties that allows us to develop a method of boundary variation suitable for waveguide grating couplers based on the method for periodic dielectric interfaces. ${ }^{8}$

We restrict ourselves to the analysis of TE-polarized, monochromatic fields for which the $E$ field remains parallel to the grating, $\mathbf{E}=\mathbf{E}_{y} \hat{\mathbf{y}}$, and therefore is determined by a single scalar quantity $u=u(z, x)$ that satisfies the homogeneous Helmholtz equation

$$
\Delta u+k^{2} u=0,
$$

where $k$ is the local wave number. We distinguish between the field that is radiated into the free space above the grating coupler, $u^{+}$, and the field radiated downward into the waveguide, $u^{-}$, as illustrated in Fig. 2.

We furthermore assume that the incident field is given by the fundamental $\mathrm{TE}$ mode for the unperturbed thinfilm waveguide. For a multilayer waveguide the field in each individual layer is given in the form

$$
E_{i}=A_{i} \exp \left(i k_{i} x-i \beta z\right)+B_{i} \exp \left(-i k_{i} x-i \beta z\right),
$$

where $k_{i}$ is given by

$$
k_{i}{ }^{2}+\beta^{2}=n_{i}{ }^{2} k_{0}{ }^{2},
$$

where $k_{0}$ is the free-space wave number and $\beta$ is the propagation constant determined by the layer thicknesses and the refractive indices. ${ }^{10} \quad A_{i}$ and $B_{i}$ in Eq. (2) are constants, which are given once the propagation constant is calculated.

The TM case can be solved in a similar way by use of $H_{x}$ rather than $E_{y}$. Although the boundary conditions will be different, the extension to the TM case is straightforward.

\section{A. Numerical Algorithm}

Let us now consider the case where the boundary between the top cladding layer and free space above the waveguide is perturbed in a way described by the function $f$ and a real number $\delta$ such that

$$
x=f_{\delta}(z)=\delta f(z)
$$

describes the upper surface of the top cladding layer. Assuming that the incoming field takes the form of Eq. (2), the radiation fields $u^{+}$and $u^{-}$must satisfy the continuity conditions

$$
\begin{aligned}
u^{+}-u^{-}= & A_{0} \exp \left[i k_{0} \delta f(z)-i \beta z\right] \\
& +B_{0} \exp \left[-i k_{0} \delta f(z)-i \beta z\right] \\
& -A_{1} \exp \left[i k_{1} \delta f(z)-i \beta z\right] \\
& -B_{1} \exp \left[-i k_{1} \delta f(z)-i \beta z\right], \\
\frac{\partial u^{+}}{\partial n}-\frac{\partial u^{-}}{\partial n}= & \frac{\partial}{\partial n}\left\{A_{0} \exp \left[i k_{0} \delta f(z)-i \beta z\right]\right. \\
& \left.+B_{0} \exp \left[-i k_{0} \delta f(z)-i \beta z\right]\right\} \\
& \times \frac{\partial}{\partial n}\left\{-A_{1} \exp \left[i k_{1} \delta f(z)-i \beta z\right]\right. \\
& \left.-B_{1} \exp \left[-i k_{1} \delta f(z)-i \beta z\right]\right\},
\end{aligned}
$$

at $x=\delta f(z)$. By requiring that the field vanish for $x$ $\rightarrow \infty$, we have $A_{0}=0$.

The central idea underlying the BV method is that the radiated fields $u^{+}$and $u^{-}$can be expanded in powers of $\delta$ :

$$
u^{ \pm}(z, x, \delta)=\sum_{n=0}^{\infty} u_{n}^{ \pm}(z, x) \delta^{n} .
$$

Bruno and Reitich established the validity of this powerseries expansion, which is a result of $u^{ \pm}$being analytic in its variables. ${ }^{11} u$, because it is a solution to the Helmholtz equation, can be expanded in a Rayleigh series:

$$
u^{ \pm}(z, x, \delta)=\sum_{r=-\infty}^{\infty} B_{r}^{ \pm}(\delta) \exp \left( \pm i \alpha_{r}^{ \pm} x-i \beta_{r} z\right),
$$

and likewise for $u_{n}^{ \pm}$:

$$
u_{n}^{ \pm}(z, x)=\sum_{r=-\infty}^{\infty} d_{n, r}^{ \pm} \exp \left( \pm i \alpha_{r}^{ \pm} x-i \beta_{r} z\right) .
$$

Given

$$
d_{n, r}^{ \pm}=\frac{1}{n !} \frac{\mathrm{d}^{n} B_{r}^{ \pm}}{\mathrm{d} \delta^{n}}(0),
$$

we recover the power-series expansion

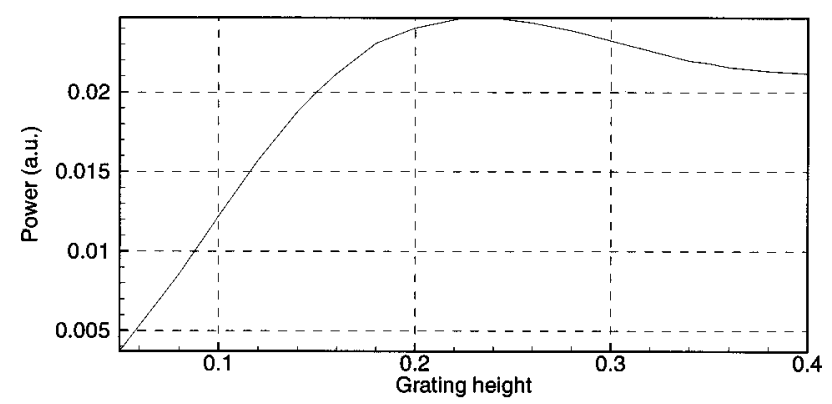

Fig. 2. Power in the -1 st diffraction order as a function of grating depth for a grating period of 0.7036 corresponding to perpendicular diffraction outcoupling. 


$$
B_{r}^{ \pm}(\delta)=\sum_{n=0}^{\infty} d_{n, r}^{ \pm} \delta^{n}
$$

for Rayleigh coefficients $B_{r}^{ \pm}$. To obtain the coefficients in this power-series expansion, we need the Fourier expansions

$$
\frac{f^{l}(z)}{l !}=\sum_{r=-l F}^{l F} C_{l, r} \exp (i K r x)
$$

for $f^{l} / l$ ! for $l$ up to some $N$. In Eq. (12), $K$ represents the smallest grating vector that we wish to resolve. For a periodic cosine surface relief, $K$ is simply the grating wave number, $K=2 \pi / \Lambda$, where $\Lambda$ is the period of the grating. For that case, the Fourier expansions need extend only to $F=1$. In the case of an aperiodic grating of finite length, $K=2 \pi / L$, where $L$ is the total length of the computational domain.

The wave vectors for the diffracted fields are given by

$$
\begin{aligned}
\beta_{r} & =\beta+r K, \\
\left(\alpha_{r}^{ \pm}\right)^{2}+{\beta_{r}}^{2} & =\left(k_{i}\right)^{2},
\end{aligned}
$$

where $i=0$ for $\alpha_{r}^{+}$and $i=1$ for $\alpha_{r}^{-}$. Only a finite number of Rayleigh modes $B_{r}^{ \pm}$will be propagating because $\alpha_{r}$ will have nonzero imaginary parts for large $r$.

The derivation of the recursive expressions for $d_{n, r}$ is thoroughly described in Ref. 8 for diffraction at an interface between two dielectrics. Here we need to modify the original derivation by using the boundary conditions for a grating coupler, Eqs. (5) and (6).

To proceed, we perform an $n$-times differentiation of the boundary conditions, Eqs. (5) and (6), with respect to $\delta$ and evaluate the result at $\delta=0$. For Eq. (5) we therefore get

$$
\begin{array}{r}
\sum_{k=0}^{n} \frac{f(z)^{n-k}}{(n-k) !}\left[\frac{\partial^{n-k}}{\partial x^{n-k}}\left(\frac{1}{k !} \frac{\partial^{k} u^{+}}{\partial \delta^{k}}\right)(z, 0,0)\right. \\
\left.-\frac{\partial^{n-k}}{\partial x^{n-k}}\left(\frac{1}{k !} \frac{\partial^{k} u^{-}}{\partial \delta^{k}}\right)(z, 0,0)\right] \\
=\frac{1}{n !}\left[-B_{0}\left(i k_{0}\right)^{n}+A_{1}\left(-i k_{1}\right)^{n}\right. \\
\left.+B_{1}\left(i k_{1}\right)^{n}\right] f(z)^{n} \exp (-i \beta z) .
\end{array}
$$

With

$$
n_{\delta}=\frac{1}{\left(1+\delta^{2} f^{\prime}(z)^{2}\right)^{1 / 2}}\left(-\delta f^{\prime}(z), 1\right),
$$

$n$ differentiations of Eq. (6) with respect to $\delta$, evaluated at $\delta=0$, yield

$$
\begin{aligned}
\sum_{k=0}^{n} & \frac{f(z)^{n-k}}{(n-k) !}\left[\frac{\partial^{n-k+1}}{\partial x^{n-k+1}}\left(\frac{1}{k !} \frac{\partial^{k} u^{+}}{\partial \delta^{k}}\right)(z, 0,0)\right. \\
& \left.-\frac{\partial^{n-k+1}}{\partial x^{n-k+1}}\left(\frac{1}{k !} \frac{\partial^{k} u^{-}}{\partial \delta^{k}}\right)(z, 0,0)\right] \\
& -\sum_{k=0}^{n-1} \frac{f^{\prime}(z) f(z)^{n-k-1}}{(n-k-1) !}\left[\frac{\partial^{n-k}}{\partial x^{n-k-1} \partial z}\left(\frac{1}{k !} \frac{\partial^{k} u^{+}}{\partial \delta^{k}}\right)(z, 0,0)\right.
\end{aligned}
$$

$$
\begin{aligned}
& \left.-\frac{\partial^{n-k}}{\partial x^{n-k-1} \partial z}\left(\frac{1}{k !} \frac{\partial^{k} u^{-}}{\partial \delta^{k}}\right)(z, 0,0)\right] \\
& =\frac{1}{n !}\left\{-B_{0}\left[i \beta n\left(i k_{0}\right)^{n-1} f^{\prime}(z) f(z)^{n-1}+\left(i k_{0}\right)^{n+1} f(z)^{n}\right]\right. \\
& \quad+A_{1}\left[i \beta n\left(-i k_{1}\right)^{n-1} f^{\prime}(z) f(z)^{n-1}-\left(-i k_{1}\right)^{n+1} f(z)^{n}\right] \\
& \left.\quad+B_{1}\left[i \beta n\left(i k_{1}\right)^{n-1} f^{\prime}(z) f(z)^{n-1}+\left(i k_{1}\right)^{n+1} f(z)^{n}\right]\right\} \\
& \quad \times \exp (-i \beta z) .
\end{aligned}
$$

From Eq. (7) we recover

$$
u_{k}^{ \pm}(z, x)=\frac{1}{k !} \frac{\partial^{k} u^{ \pm}}{\partial \delta^{k}}(z, x, 0)
$$

such that Eqs. (15) and (17) give

$$
\begin{aligned}
u_{n}^{+}- & u_{n}^{-} \\
= & \frac{1}{n !}\left[-B_{0}\left(i k_{0}\right)^{n}+A_{1}\left(-i k_{1}\right)^{n}+B_{1}\left(i k_{1}\right)^{n}\right] f(z)^{n} \\
& \times \exp (-i \beta z)-\sum_{k=0}^{n-1} \frac{f(z)^{n-k}}{(n-k) !}\left(\frac{\partial^{n-k} u_{k}^{+}}{\partial x^{n-k}}-\frac{\partial^{n-k} u_{k}^{-}}{\partial x^{n-k}}\right), \\
\frac{\partial u_{n}^{+}}{\partial x}- & \frac{\partial u_{n}^{-}}{\partial x} \\
= & \frac{1}{n !}\left\{-B_{0}\left[i \beta n\left(i k_{0}\right)^{n-1} f^{\prime}(z) f(z)^{n-1}+\left(i k_{0}\right)^{n+1} f(z)^{n}\right]\right. \\
& +A_{1}\left[i \beta n\left(-i k_{1}\right)^{n-1} f^{\prime}(z) f(z)^{n-1}-\left(-i k_{1}\right)^{n+1} f(z)^{n}\right] \\
& +B_{1}\left[i \beta n\left(i k_{1}\right)^{n-1} f^{\prime}(z) f(z)^{n-1}\right. \\
& \left.\left.+\left(i k_{1}\right)^{n+1} f(z)^{n}\right]\right\} \exp (-i \beta z) \\
& +\sum_{k=0} \frac{f^{\prime}(z) f(z)^{n-k-1}}{(n-k-1) !}\left(\frac{\partial^{n-k} u_{k}^{+}}{\partial x^{n-k-1} \partial z}-\frac{\partial^{n-k} u_{k}^{-}}{\partial x^{n-k-1} \partial z}\right) \\
& -\sum_{k=0}^{n-1} \frac{f(z)^{n-k}}{(n-k) !}\left(\frac{\partial^{n-k+1} u_{k}^{+}}{\left.\partial x^{n-k+1}-\frac{\partial^{n-k+1} u_{k}^{-}}{\partial x^{n-k+1}}\right) .},\right.
\end{aligned}
$$

By substituting the Rayleigh expansions for $u_{n}^{ \pm}$[Eq. (9)] into Eq. (19), we recover the coefficients $d_{n, r}$ from a recurrence in $d_{k, r}, k<n$, and from the Fourier coefficients $C_{k, r}$, in the form

$$
\begin{aligned}
\sum_{r=-\infty}^{\infty}( & \left.d_{n, r}^{+}-d_{n, r}^{-}\right) \exp \left(-i \beta_{r} z\right) \\
= & {\left[-B_{0}\left(i k_{0}\right)^{n}+A_{1}\left(-i k_{1}\right)^{n}+B_{1}\left(i k_{1}\right)^{n}\right] } \\
& \times \sum_{r=-n F}^{n F} C_{n, r} \exp \left(-i \beta_{r} z\right) \\
& -\sum_{k=0}^{n-1}\left[\sum_{r=-(n-k) F}^{(n-k) F} C_{n-k, r} \exp (i K r z)\right] \\
& \times\left\{\sum _ { r = - \infty } ^ { \infty } \left[\left(i \alpha_{r}^{+}\right)^{n-k} d_{k, r}^{+}\right.\right. \\
& \left.\left.-\left(-i \alpha_{r}^{-}\right)^{n-k} d_{k, r}^{-}\right] \exp \left(-i \beta_{r} z\right)\right\} .
\end{aligned}
$$


In a similar fashion, substituting Eq. (9) into Eq. (20) yields

$$
\begin{aligned}
& \sum_{r=-\infty}^{\infty}\left(i \alpha_{r}^{+} d_{n, r}^{+}+i \alpha_{r}^{-} d_{n, r}^{-}\right) \exp \left(-i \beta_{r} z\right) \\
& =\sum_{r=-n F}^{n F} C_{n, r}\left\{-B_{0}\left[i \beta\left(i k_{0}\right)^{n-1}(i K r)+\left(i k_{0}\right)^{n+1}\right]\right. \\
& +A_{1}\left[i \beta\left(-i k_{1}\right)^{n-1}(i K r)-\left(-i k_{1}\right)^{n+1}\right] \\
& \left.+B_{1}\left[i \beta\left(i k_{1}\right)^{n-1}(i K r)+\left(+i k_{1}\right)^{n+1}\right]\right\} \exp \left(-i \beta_{r} z\right) \\
& +\sum_{k=0}^{n-1}\left[\sum_{r=-(n-k) F}^{(n-k) F} C_{n-k, r} i K r \exp (i K r z)\right] \\
& \times\left\{\sum _ { r = - \infty } ^ { \infty } \left[\left(i \alpha_{r}^{+}\right)^{n-k-1}\left(-i \beta_{r}\right) d_{k, r}^{+}\right.\right. \\
& \left.\left.-\left(-i \alpha_{r}^{-}\right)^{n-k-1}\left(-i \beta_{r}\right) d_{k, r}^{-}\right] \exp \left(-i \beta_{r} z\right)\right\} \\
& -\sum_{k=0}^{n-1}\left[\sum_{r=-(n-k) F}^{(n-k) F} C_{n-k, r} \exp (i K r x)\right] \\
& \times\left\{\sum _ { r = - \infty } ^ { \infty } \left[\left(i \alpha_{r}^{+}\right)^{n-k+1} d_{k, r}^{+}\right.\right. \\
& \left.\left.-\left(-i \alpha_{r}^{-}\right)^{n-k+1} d_{k, r}^{-}\right] \exp \left(-i \beta_{r} z\right)\right\} .
\end{aligned}
$$

After some manipulations, utilizing the fact that $d_{k, q}^{ \pm}$ $=0$ for $|q|>k F$, we recover following recursive formulas for the coefficients $d_{n, r}^{ \pm}$:

$$
\begin{aligned}
d_{n, r}^{+}-d_{n, r}^{-} & \\
= & {\left[-B_{0}\left(i k_{0}\right)^{n}+A_{1}\left(-i k_{1}\right)^{n}+B_{1}\left(i k_{1}\right)^{n}\right] C_{n, r} } \\
& -\sum_{k=0}^{n-1} \sum_{q=\max [-k F, r-(n-k) F]}^{\min [k F, r+(n-k) F]} C_{n-k, r-q}\left[\left(i \alpha_{q}^{+}\right)^{n-k} d_{k, q}^{+}\right. \\
& \left.-\left(-i \alpha_{q}\right)^{n-k} d_{k, q}^{-}\right], \\
i \alpha_{r}^{+} d_{n, r}^{+}+ & i \alpha_{r}^{-} d_{n, r}^{-} \\
=\{ & -B_{0}\left(i k_{0}\right)^{n-1}\left[\beta K r+\left(i k_{0}\right)^{2}\right] \\
& +A_{1}\left(-i k_{1}\right)^{n-1}\left[\beta K r+\left(-i k_{1}\right)^{2}\right] \\
& \left.+B_{1}\left(i k_{1}\right)^{n-1}\left[\beta K r+\left(i k_{1}\right)^{2}\right]\right\} C_{n, r} \\
& n-1 \quad \min [k F, r+(n-k) F] \\
& +\sum_{k=0} \sum_{q=\max [-k F, r-(n-k) F]} C_{n-k, r-q}\{[i K(r-q)] \\
& \times\left(-i \beta_{q}\right)\left[\left(i \alpha_{q}^{+}\right)^{n-k-1} d_{k, q}^{+}-\left(-i \alpha_{q}^{-}\right)^{n-k-1} d_{k, q}^{-}\right] \\
& \left.-\left[\left(i \alpha_{q}^{+}\right)^{n-k+1} d_{k, q}^{+}-\left(-i \alpha_{q}^{-}\right)^{n-k+1} d_{k, q}^{-}\right]\right\} .
\end{aligned}
$$

Once the power series expansion coefficients $d_{n, r}^{ \pm}$are determined, the Rayleigh expansion coefficients $B_{r}^{ \pm}$ can be computed from the power series, Eq. (11). The radius of convergence, however, of this Taylor series is rather small. To overcome this limitation we recast, as suggested in Ref. 8, the expansion as a Padé approximation, which significantly enhances the radius of convergence. We find that, in general, using an $[M / M]$ approximant, i.e., using the same order of polynomial in the numerator as in the denominator, yields the fastest convergence for our problem. As for the computation of the Fourier spectrum of the surface relief $f(z)$ [Eq. (12)], we use the fast Fourier transform for enhanced computational speed.

It is important to observe that for the analysis of grating couplers the BV method is approximate, as it does not account for the attenuation of the guided wave caused by the loss of energy by coupling to free space. For grating couplers of moderate length this lack of precision is not believed to be a serious problem, since the overall diffraction efficiency is typically less than 5\%. However, if highly diffracting structures were to be analyzed, a feedback mechanism could possibly be built into the model.

Another approximation lies in the fact that the method does not account for any reflections at lower-lying boundaries of the downward radiation that is also subsequently coupled to free space. As we shall demonstrate, however, the method provides highly accurate solutions to simple as well as to nontrivial test problems.

\section{B. Postprocessing}

In principle, the diffracted field can be recovered anywhere above the grating coupler from the Rayleigh series expansion [Eq. (8)] directly. However, because of the periodicity that is inherently assumed in the formulation of the scheme, we find it more convenient to evaluate the diffractive field along an aperture covering the grating coupler and to use this field to recover the near- and farfield radiation from the structure through the use of the surface-equivalence theorem. ${ }^{12}$ To maintain high accuracy we compute the diffracted field on a set of quadrature points on which high-order integration can be performed.

\section{ANALYSIS OF PERIODIC GRATING COUPLERS}

In the following, we shall demonstrate the convergence of the proposed scheme for purely periodic gratings of infinite extent and give further examples of the analysis facilitated by the proposed method. Below, all waveguide dimensions as well as length parameters are normalized to the free-space wavelength $\lambda$ of the incident field.

As the basic waveguide parameters for the numerical examples, we use values that are representative of optical materials such as glass and polymers. We consider a waveguide structure consisting of a core layer with refractive index $n=1.45$ and thickness $d_{1}=0.8$ sandwiched between two cladding layers of refractive index $n=1.4$. The top cladding layer has a finite thickness $d_{2}=1$, and above this layer is air with $n=1$. For the fundamental TE mode this geometry yields an effective index of 1.4213. 
We consider a cosine surface relief

$$
f_{\delta}(z)=A \cos \left(\frac{2 \pi}{\Lambda} z\right)
$$

Let us first study the convergence of the scheme. As we do not have an analytic solution to compare with, we are unable to compute the error. Rather, we look at the power coupled to the -1 st diffraction order as we increase the number of terms in the Padé approximation to the Taylor series expansion [Eq. (11)]. Table 1 confirms the convergence in the case of $A=0.1$ as the number of terms in the power series expansion increases.

To investigate the sensitivity of the convergence further, we consider the convergence for increasing amplitudes of the surface relief. As we increase $A$, the error of the scheme increases in the sense that the number of significant digits for $\bar{P}$ decreases. To give an estimate of the error, we compute the average and the standard deviation for $M$ ranging from 15 to 49 for increasing $A$, as shown in Table 2, which illustrates that, as we increase the amplitude of the surface relief, the standard deviation increases. However, even for an amplitude of 0.4, yielding a height-to-period ratio of the surface relief of more than 1 , the solution remains bounded with a reasonable error. We find that, when the amplitude is increased beyond 0.4, the scheme fails to converge.

It is worthwhile making another observation from Table 2, which clearly shows that there appears to be a maximum for the radiation into the -1 st diffraction order. To analyze this, we show in Fig. 3 the power in the -1 st order as a function of grating height, which confirms

Table 1. Power in the -1 st Diffraction Order for Different Numbers of Terms $[M, M]$ in the Padé Approximation to the Power-Series Expansion

\begin{tabular}{rc}
\hline$M$ & $\bar{P}\left(\times 10^{-2}\right)$ \\
\hline 2 & 1.208589 \\
3 & 1.218618 \\
4 & 1.220834 \\
5 & 1.220240 \\
6 & 1.220247 \\
7 & 1.220242 \\
8 & 1.220243 \\
9 & 1.220243 \\
10 & 1.220243 \\
\hline
\end{tabular}

Table 2. Convergence for Periodic Grating Coupler Analysis ${ }^{a}$

\begin{tabular}{lll}
\hline$A$ & $\bar{P}\left(\times 10^{-2}\right)$ & \multicolumn{1}{c}{$\sigma$} \\
\hline 0.1 & 1.220243 & $8 \times 10^{-7}$ \\
0.2 & 2.410162 & $6 \times 10^{-6}$ \\
0.3 & 2.3120 & 0.004 \\
0.4 & 2.14 & 0.11 \\
\hline
\end{tabular}

${ }^{a} \bar{P}$ is the average power in the -1 st diffraction order and $\sigma$ is the standard deviation over the length of the power series ranging from 15 to 49 .
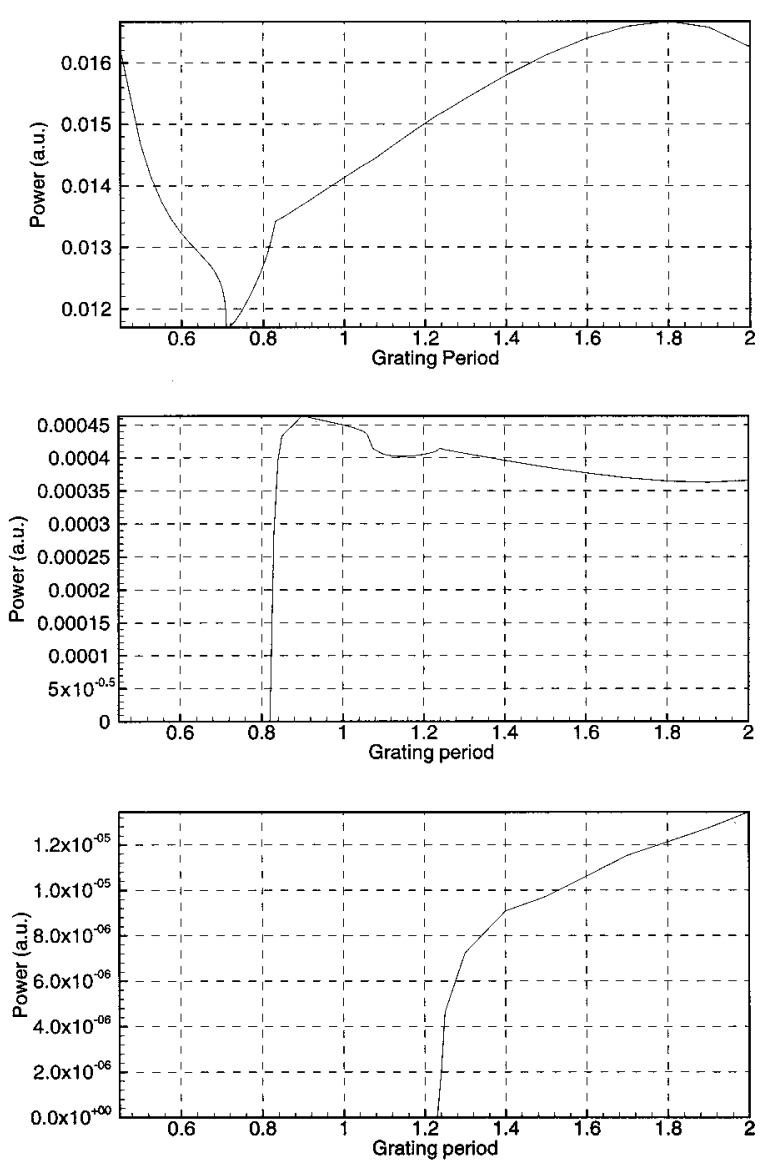

Fig. 3. Power output in (top) -1 st, (center) -2 nd, and (bottom) -3 rd diffraction orders as a function of grating period $\Lambda$ of the surface relief.

that, as expected, more power is radiated when the height of the surface-relief grating is increased. However, the power has a maximum at a grating height of 0.23 above which the grating coupler becomes less efficient. A possible explanation for this is that, when the surface relief becomes deep, the propagation of the guided wave in the thin-film waveguide becomes heavily disturbed by the surface relief, leading to a reduced coupling to the -1 st diffraction order. Certainly, as we shall see for the FGC, the near-field radiation from a deep surface relief is severely distorted.

As another example we keep a fixed amplitude $A$ $=0.1$ and consider the output power in the diffraction orders as a function of the grating period, $\Lambda$. Figure 3 shows the power output in three diffraction orders, $m$ $=-1,-2$, and -3 , as a function of the grating period, and a number of things are worth noticing. First, we see that the -1 st diffraction order has a global minimum for a grating period of $\Lambda=1 / 1.4213$, corresponding to a perpendicular output. This is not surprising from a physical point of view because of the energy conservation. A second observation to be made is that the appearance of a second diffraction order leads to an abrupt change in the slope of the curve of the first diffraction order. A similar change of slope is seen for the -2 nd diffraction order at the cutoff for the -3 rd diffraction order. 


\section{FOCUSING GRATING COUPLERS}

Let us now demonstrate the use of the proposed BV method to study aperiodic surface-relief gratings couplers of finite length. Clearly, as we use a discrete Fourier series expansion for the surface relief, the grating is implicitly forced to be periodic. However, as we shall demonstrate, when a sufficiently large total length of the computational domain relative to the length of the finite surface relief is chosen, the results become consistent with those obtained by a method that deals with truly finite gratings.

For the FGC surface relief we use the generic profile

$$
\begin{aligned}
f_{\delta}(z)= & A \exp \left[-\left(\frac{z-z_{0}}{w}\right)^{2}\right] \\
& \times \cos \left\{2 \pi\left[a_{0}+a_{1}\left(z-z_{0}\right)\right]\left(z-z_{0}\right)\right\},
\end{aligned}
$$

where $A$ is the amplitude, $w$ is the width of the exponentially truncated relief, $z_{0}$ is the center of the relief, $a_{0}$ $=1 / \Lambda$ for the unchirped relief, and $a_{1}$ is the chirp parameter.

To establish the necessary length to simulate a FGC of finite extent we investigate the far-field radiation pattern when varying the total length $L$ of the computation domain. The number of modes in the Fourier transform is scaled with $L$ to maintain a constant resolution. Figure 4 shows the far-field radiation pattern for four values of $L$

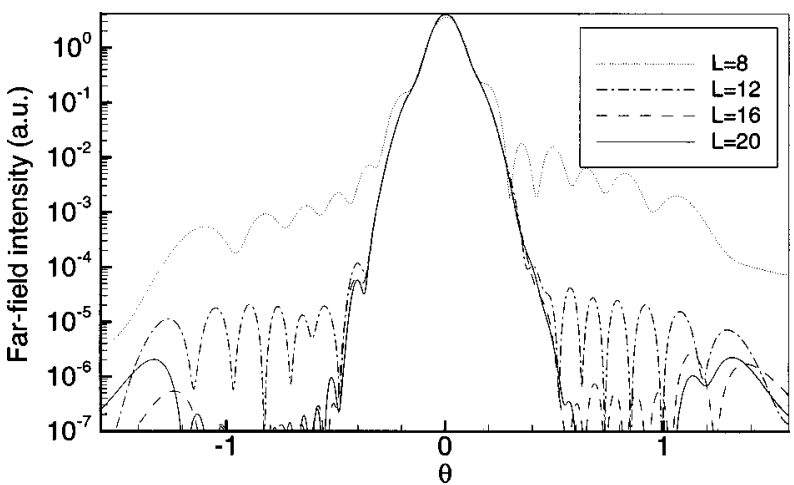

Fig. 4. Far-field radiation pattern for four lengths, $L$, of the computational domain.

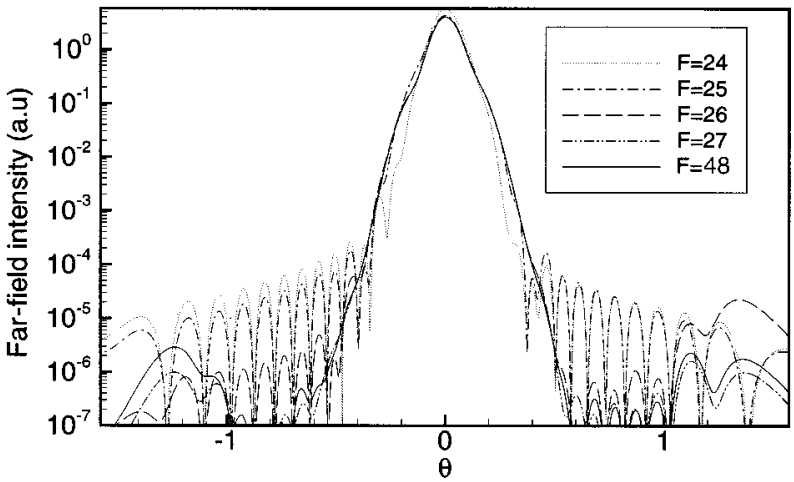

Fig. 5. Far-field radiation pattern for various resolutions used in the Fourier series [Eq. (12)] to represent the surface relief. $F$, number of Fourier modes (24-48 here).

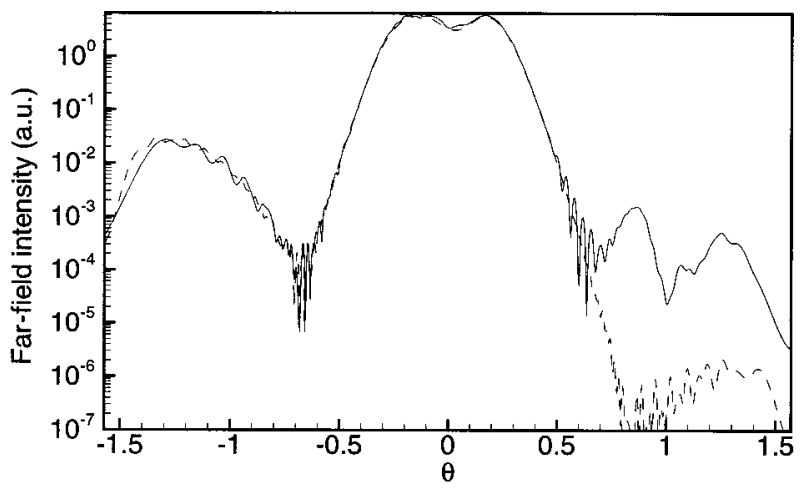

Fig. 6. Far-field radiation patterns for a FGC computed by the BV method (dashed curve) and by the SC method (solid curve).

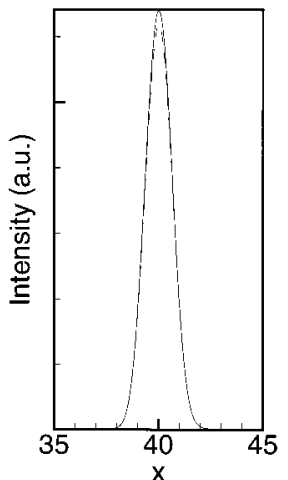

(a)

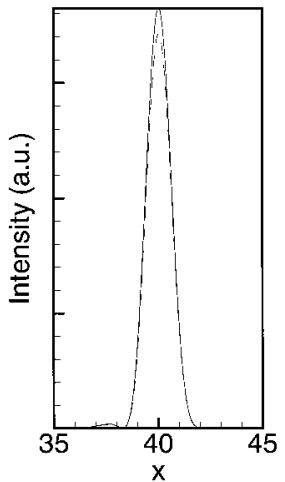

(b)

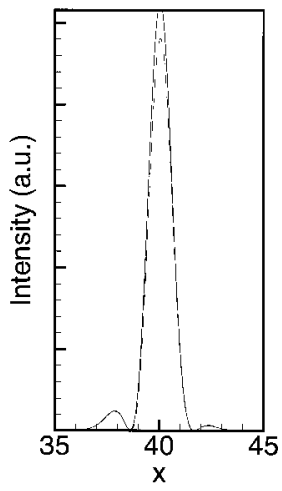

(c)
Fig. 7. Near-field radiation patterns for surface-relief amplitudes (a) 0.1 , (b) 0.2 , and (c) 0.3 by the BV method (dashed curves) and the SC (solid curves) methods.

Table 3. Key Figures for SC and Proposed BV Computations $^{a}$

\begin{tabular}{cccccrr}
\hline & & $M(\mathrm{BV})$ & \multicolumn{2}{c}{$f$} & & \multicolumn{2}{c}{ Time } \\
\cline { 3 - 4 } \cline { 6 - 7 }$A$ & $\mathrm{BV}$ & $\mathrm{BV}$ & $\mathrm{SC}$ & & BV (s) & SC (h) \\
\hline 0.1 & 7 & 47.1 & 47.0 & & 46 & 30 \\
0.2 & 13 & 46.3 & 46.2 & & 390 & 64 \\
0.3 & 17 & 45.2 & 45.1 & & 1081 & 126 \\
\hline
\end{tabular}

${ }^{a} A$ is the amplitude of the surface relief. An $[M, M]$ Padé approximant is used for the BV method. $f$ is the focal length normalized with the free-space wavelength, and Time means the total computation time.

with the following parameters for the surface relief: $A$ $=0.25, a_{0}=1.4213, a_{1}=0.005$, and $w=3$. From the figure it is clear that, once the length of the computation domain exceeds 16 , or $\sim 5 w$, the sidelobes caused by the periodicity are efficiently suppressed.

Another issue related to the Fourier transform is the resolution required for accurately resolving the structure of the surface relief, i.e., the number of terms in Eq. (12). To address this issue we performed a number of simulations with several resolutions for the same geometry as discussed above. The results are illustrated in Fig. 5, where we find that once the number of Fourier modes exceeds $F=26$, corresponding to a little more than two 
modes per grating period for this case, the sidelobes are efficiently suppressed and the center lobe well resolved. It should be noted that the Fourier spectrum of the surface relief is affected by both the width of the Gaussian truncation and the chirp of the grating period, so a higher resolution may be necessary for other values of these parameters.

Having established resolution and computationaldomain requirements for our problem, let us perform a direct comparison with a highly accurate spectral collocation (SC) method. ${ }^{6}$ This method computes a rigorous solution of the vectorial time-domain Maxwell equations, and our group has previously demonstrated its superior accuracy. ${ }^{13}$

For the comparison, we study a longer FGC. The total length of the computation domain is now $80 \lambda, w=12 \lambda$, $a_{0}=1.4213, a_{1}=0.01$, and $A=0.3$, yielding a heightto-period ratio close to 1 . As we can see from Fig. 6, there is excellent agreement between the far-field patterns of the two methods, even for this relatively deep surface relief grating.

To further validate the proposed approach, we compute a number of solutions, using both the BV and the SC methods, and compare them with the near-field solutions. Figure 7 shows a line scan of the intensity in the focal plane for both methods for three values of the surface relief amplitude, and Table 3 lists key figures for the simulations. The computations were performed on a singleprocessor Sun Ultra-1 workstation.

We find that the excellent correspondence in the far field is maintained in the near field even though the intensity is slightly lower for the BV method for all amplitudes, which may be due to not accounting for multiple reflections. We also confirm that the use of deep surface reliefs leads to a deteriorated outcoupling from the waveguide grating coupler: While it is clear that for $A=0.1$ the intensity is symmetric and Gaussian in the focal plane, we find that as the amplitude increases to $A$ $=0.2$ and $A=0.3$, sidelobes evolve and an asymmetry becomes noticeable. We also see a shift in the focal plane (Table 3) toward the surface relief for both methods, which agree well on the focal length.

To illustrate the reduction in computation time that is achievable by the BV method, in Table 3 we also list computation times obtained with the two methods. For the BV method the computation time increases with the depth of the surface-relief grating, as it is necessary to go to higher order in the power series expansion of the Rayleigh coefficients. The time listed for the SC method is the computation time necessary for reaching a steadystate solution for this time-domain method, after which the steady-state near- and far-field radiation can be computed. Again, as the surface relief gets deeper, it takes longer for the problem to reach steady state, hence the increasing computation time. Comparing computation times in Table 3, we can see that the use of the approximate BV method certainly pays off: While we find excellent agreement with the rigorous SC method, we find that a reduction in the computation time by more than a factor of 2000 is achievable, a fact that calls for use of the method in the future as the forward solver in an optimization scheme.

\section{CONCLUSIONS}

We have presented the development of a boundary variation (BV) method for the analysis of both periodic and aperiodic waveguide grating couplers and have given examples of the analysis of continuous surface-relief gratings. For a periodic grating we have found that the power radiated into the fundamental -1 st diffraction order does not increase monotonically with the grating height. Rather, an optimum exists, and we attribute this to the distortion of the guided-wave propagation that results from very deep surface reliefs.

For a focusing grating coupler we have found excellent agreement with the highly accurate spectral collocation method. A reduction in computation time of up to more than 2000 times compared with a rigorous approach is achieved.

These highly encouraging results suggest that further development along the lines discussed here are worthwhile, and we are currently considering the formulation of the BV-based methods for the three-dimensional forward-scattering problem.

\section{ACKNOWLEDGMENTS}

We gratefully acknowledge fruitful discussions on the BV method with Oscar Bruno and Fernando Reitich. J. S. Hesthaven acknowledges partial support for this study from Defense Advanced Research Projects Agency/U.S. Air Force Office of Scientific Research grant F49620-10426.

P. G. Dinesen's e-mail address is palle.dinesen @risoe.dk.

\section{REFERENCES}

1. T. K. Gaylord and M. G. Moharam, "Analysis and applications of optical diffraction by gratings," Proc. IEEE 73, 894-937 (1985)

2. B. Lichtenberg and N. C. Gallagher, "Numerical modeling of diffractive devices using the finite element method," Opt. Eng. 33, 1592-1598 (1994).

3. K. Hirayama, E. N. Glytsis, T. K. Gaylord, and D. W Wilson, "Rigorous electromagnetic analysis of diffractive cylindrical lenses," J. Opt. Soc. Am. A 13, 2219-2231 (1996).

4. D. W. Prather and S. Shi, "Formulation and application of the finite-difference time-domain method for the analysis of axially symmetric diffractive optical elements," J. Opt. Soc. Am. A 16, 1131-1141 (1999).

5. K. H. Dridi and A. Bjarklev, "Optical electromagnetic vector-field modeling for the accurate analysis of finite diffractive structures of high complexity," Appl. Opt. 38, 1668-1676 (1999)

6. J. S. Hesthaven, P. G. Dinesen, and J.-P. Lynov, "Spectral collocation time-domain modeling of diffractive optical elements," J. Comput. Phys. 155, 287-306 (1999).

7. O. P. Bruno and F. Reitich, "Numerical solution of diffraction problems: a method of variation of boundaries," J. Opt. Soc. Am. A 10, 1168-1175 (1993).

8. O. P. Bruno and F. Reitich, "Numerical solution of diffraction problems: a method of variation of boundaries. II. Finitely conducting gratings, Padé approximants, and singularities," J. Opt. Soc. Am. A 10, 2307-2316 (1993).

9. O. P. Bruno and F. Reitich, "Numerical solution of diffraction problems: a method of variation of boundaries. III. Doubly periodic gratings," J. Opt. Soc. Am. A 10, 25512562 (1993). 
10. S. Ramo, J. R. Whinnery, and T. van Duzer, Fields and Waves in Communications Electronics, 3rd ed. (Wiley, New York, 1993).

11. O. Bruno and F. Reitich, "Solution of a boundary value problem for Helmholtz equation via variation of the boundary into the complex domain," Proc. R. Soc. Edinburgh, Sect. A: Math. 122, 317-340 (1992).
12. S. A. Schelknuoff, "Some equivalence theorems of electromagnetics and their application to radiation problems," Bell Syst. Tech. J. 15, 92-112 (1936).

13. P. G. Dinesen, J. S. Hesthaven, J. P. Lynov, and L. Lading, "Pseudo-spectral method for the analysis of diffractive optical elements,” J. Opt. Soc. Am. A 26, 1124-1130 (1999). 